\title{
Research and Practice on the Dynamic Design of Visualized Image Identity for Art Exhibition
}

\author{
Dapeng Zhou \\ School of Art and Design \\ Guangdong Industry Polytechnic \\ Guangzhou, China
}

\begin{abstract}
This paper has elaborated the dynamic tendency of visual images on art exhibition with new media as the technical support under the historical background of "Internet Plus" and proposed the systematic design methods and strategies, in order to build the identification system of visual images that conforms to the dissemination environment of new media. Meanwhile, the project on the art design exhibition hosted by the author has provided practice verification and application support for the above theory.
\end{abstract}

Keywords - Internet; new media; exhibition on visual identity design; dynamic symbol

\section{INTRODUCTION}

Dynamic visual design is a unique visual expression form of art produced in the rapid development of new media technology when we enter the 21 st century. With the continuous production of new media in the information era, the dissemination platform of information has expanded from the traditional paper media to electronic screen, having produced the new demands of dynamic design. The dynamic design has been extensively applied to fields such as the opening of films and television, interactive media, public art and space arts. The form of visual communication design can show the aesthetic feelings of 4D space design beyond the reach of the traditional $2 \mathrm{D}$ or $3 \mathrm{D}$ forms, and it is the key feature of new prominent visual communication in the new era. In terms of the practical application of design, capable of bringing visual shock, dynamic design in activities of commercial design has more advantages than the traditional static communication. The features of new media determine the media form and the fast-growing form change continuously. When more attentions are paid to the new media and the application is popular, the adoption of dynamic design will become a universal phenomenon in the field of visual communication design.

\section{Change OF Visual COMMUNICATION AND} COGNITIVE STYLE UNDER THE SitUATION OF "INTERNET PLUS"

The "Internet Plus" was included in the annual top 10 new words and top 10 catchwords in the Report on Language Life in China (2016) issued by the Ministry of Education and the State Language Work Committee in May 2016. [1] "Internet Plus" bases on respecting and meeting people's most basic needs and is driven by innovation. The digital information and communication technology is used to deeply integrate the internet and the traditional industries. Meanwhile, it aims at connecting everything through crossborder integration and realizing the reshape of structure eventually. Under the situation of "Internet Plus", the digital information technology has made our visual communication and cognitive style happen revolutionary reform. The delivery tools of new media that base on digital technology such as QQ, WeChat, Twitter and Facebook have changed the previous one-way mass communication mode and path through terminal devices such as computer, PAD and mobile phones, realizing that the information requirements are diversified, the target audience is subdivided, the information push is precise, the information content is multi-dimensional, the transformation and its efficiency are timely and efficient and the spreading scope is more extensive.

\section{THE DYNAMIC TREND OF THE IDENTITY DESIGN OF VISUAL IMAGES UNDER THE SITUATION OF "INTERNET PLUS"}

The increasingly strong spiritual and cultural needs of the masses have driven the consumption of art and culture. Meanwhile, art exhibition grounds and art exhibition flourish. By virtue of the advantages of digital media, the visual image design makes the dispersed visual contacts on art exhibitions form an organic whole to better interact with the society, attract more people to participate and expand the social influence of exhibition. Exhibiting visual images includes two tasks, namely "image construction" and "image release and dissemination". After visual images are established through "image construction", the images are displayed and disseminated to the target audience through various media resources to achieve the effect of spreading information. New media change simple and centralized paradigm of the traditional paper media in information spreading and have provided us with various possibilities such as dynamic changes, interactive experience and multidimensional dissemination. Therefore, visual image design of art exhibition must combine with it, which is of great importance under the historical background that media go ahead of the rest and internet is taken as the prior release medium 
Visual image design has experienced the process of evolution and development from realistic painting to abstract symbol, from complicated pattern modeling to concise graphic style. Nowadays, under the situation of "Internet Plus", the traditional plane and static visual images have been unable to meet the needs of multimedia spreading and brand competition. The dynamic presentation of visual images has gradually become an important development trend of visual image design. Ideas that cannot be completely shown in 2D spaces will be expressed through dimensions of space, time and voice and the dynamic design of figure, color, character, movement and voice. Once being released, the logo of Hannover World Expo in Germany in 2000 immediately amazed people around the world. The design of it is earthshaking and has groundbreaking significance in the history of design. Visual figures change unpredictably, embodying some uncertainty. The growth and flowing changes of figures match with key words of the age such as interaction, high speed, ecology and development, echoing with the connotation of the subject of the World Expo (see "Fig. 1 "). Hannover is to show the process with the continuous evolution of "Human, Nature and Technology" [2].

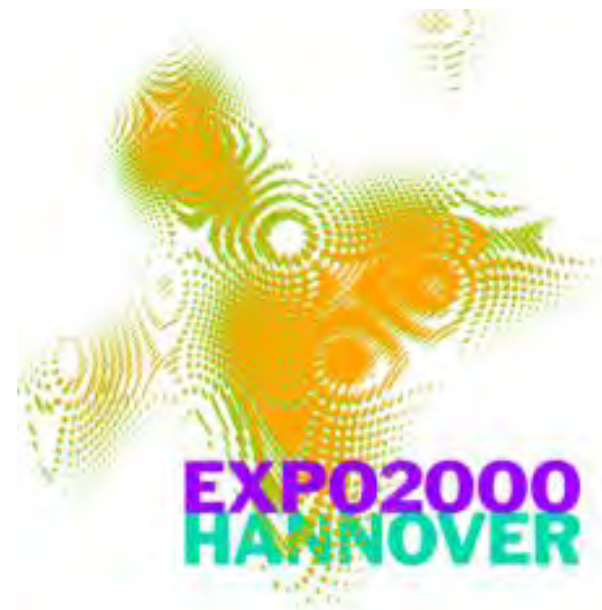

Fig. 1. Logo of Hannover World Expo in Germany in 2000.

\section{DYNAMIC DESIGN STRATEGY OF THE VisuAL IMAGE IDENTITY}

First is the design strategy that centers on the shape composition. Different from the traditional static visual images, the expression form of shape composition is not fixed. Visual images will have different forms when it is observed from different perspectives and applied to different environment. It has fully considered the possibility of the dynamic expansion of images. New design of city image was released by Melbourne Australia in 2009 (see "Fig. 2 "). The initial $\mathrm{M}$ of the English Melbourne is taken as the main visual element to reflect the diversified, innovative and habitable city images that pay attention to ecology through deconstruction and reconstruction and free changes. It is thoughtful in aspects of systematic application and media promotion. Its abundant expressive force and visual tension are easily been accepted by the public, successfully injecting this city with vitality and grace.

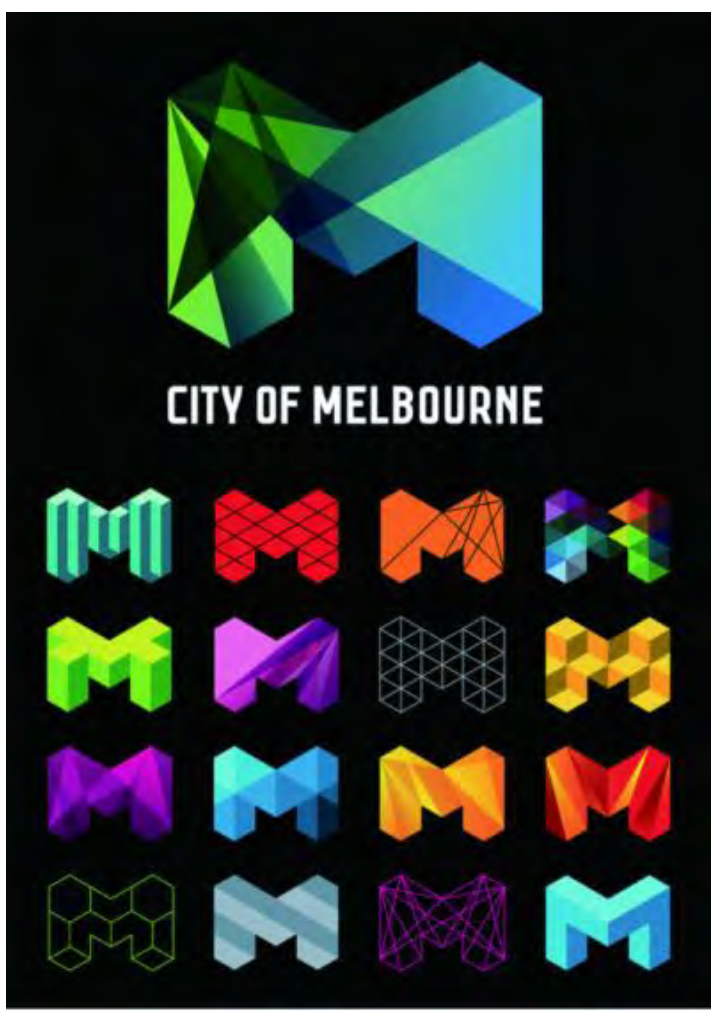

Fig. 2. System application design of new city image of Melbourne.

Second is the design strategy that centers on dynamic images. The dimension of time is added on the basis of 2D through taking advantage of digital media. And it appears in the form of dynamic images such as gif and videos. Static images are changed into dynamic images, and even narrative plots are added to make the information spreading more vivid and appealing and give people visual, auditory and tactile sense experience. The inspiration of the visual image design in the Nagasaki Prefectural Art Museum of Japan originates from the appearance of the building of the art museum. Digital technology has been used to form the abstract dynamic graphics that echo with the image of building entity (see "Fig. 3 "). These vertical bars randomly move right and left. The integration of separation and reunion and the alternation of move and motionlessness have correctly expressed the peace and quiet and elegant public image of the art museum and the visual theme that immerses in the artistic and humanistic atmosphere. 

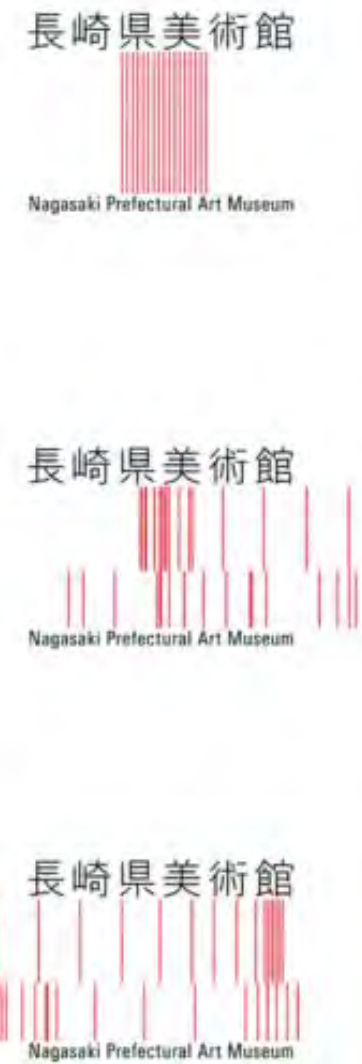

Fig. 3. Visual image design of Nagasaki Prefectural Art Museum of Japan.

Third is the design strategy that centers on the interactive experience of the audiences. The application of new technologies such as digital communication, mobile internet, computer design and virtual reality has expanded the connotation and extension of visual culture. User experience is strengthened through interactive design. In reality, it has extended the process of audiences in perceiving and experiencing visual images and information. The target audience transfer from one-way reception of information to two-way interactive experience, having strengthened the intensity and effectiveness of information spreading in the levels of experience and emotion communication. When important festivals arrive, Google will redesign the visual images according to the themes of festivals (see "Fig 4") and realize user participation in real sense. For example, on Jun.9, 2011, to memorize the 96th birthday anniversary of Les Paul, the father of electric guitar, the visual image on the home page of Google is redesigned by the elements of electric guitar (see "Fig. 5"). When the mouse of users touches on the string carelessly, each string will vibrate and produce sound. People can play the "electric guitar" through keyboard or mouse. The dynamic narrative advantages will give play to the initiative of elements of visual images and have strengthened the spreading of the whole system of visual images.
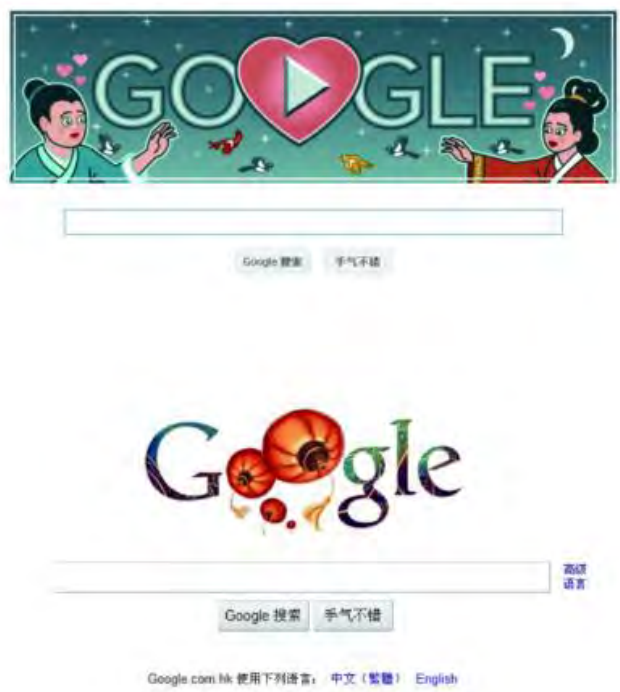

Fig. 4. Page design of GOOGLE search on the Chinese Valentine's Day and the National Holiday.
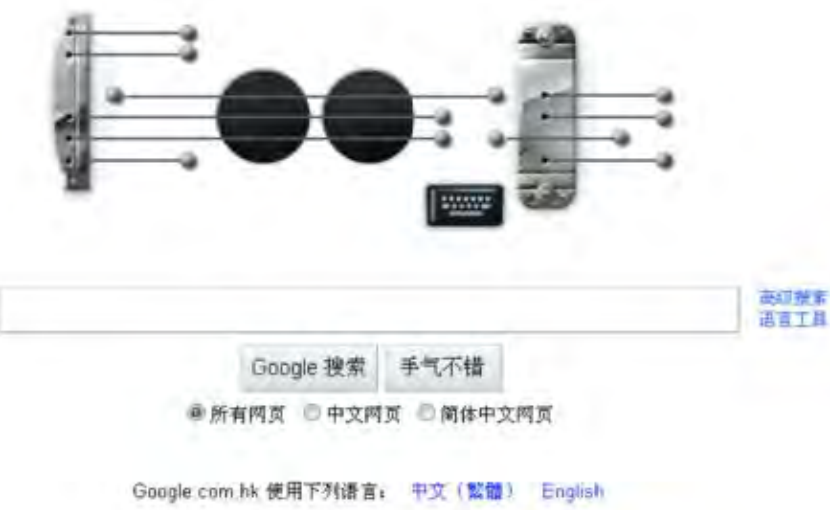

Fig. 5. Page design of Google search in memory of the father of electric guitar.

To sum up, under the current situation of "Internet Plus", designers have got rid of the design restrictions brought by the technology and materials such as the plate making and printing of traditional media, the design and production of 3D images and the installation and release of outdoor media. At the same time, it has gradually broken through the simple, concise and plane visual aesthetical features of modernism on this basis. Under the strong support of new technology, designers are free from the limitation of technology and means and give full play to their creative thinking and imagination and realize their design ideas. In addition, information is passed through multiple dimensions and ways. In the era when mobile internet has become the main media of information spreading, the interaction of screens is the future development tendency. Visual communication design has gradually established the aesthetic style of new media that takes interaction as the feature, breaks through the visual category and spans multiple dimensions and integrates 
various media effects such as static state, animation and audios. New technologies under the situation of "Internet Plus" and the characteristics and advantages of different media have been used to magnify the function of visual communication.

\section{DyNAMic Design Practice of the Visual IMAGE IDENTITY ON ART EXHIBITIONS}

Visual images must be the real expression of cultural connotation of exhibition. "Graduation design" presents the result of design education in schools in the most direct way and is the first collective exhibition that all the graduates leave the protection of schools and face the society. At present, the School of Art and Design of Guangdong Industry Polytechnic has 10 majors, 16 professional directions and more than 5,800 full-time students. It is the important base of design education in Guangdong even in South China. The yearly graduation design exhibition is regarded as a gala of the design circle in Guangdong and attracts the attentions from the Pearl River Delta Region, peer colleges, industry professionals and media as well as all sectors of society. In 2016, the graduation design exhibition walked out of the campus for the first time and was held in the Pati creative art district on the bank of the Pearl River in the center of Guangzhou City, which is praised as the landmark of fashion in South China. Entrusted by the committee of the exhibition, the author and his design team are responsible for the visual image design of the exhibition activity. The whole graduation exhibition, in the "panorama" pattern, has comprehensively shown the works of more than 1,000 groups of graduates in 16 professional directions, 10 majors and 6 professional departments including visual communication design, industrial design, environmental art design, digital media design, decoration design and costume and costume design.

The main audience of the exhibition is the professional staff of universities, scientific research institutions, enterprise and public institutions related to design in the Pearl River Delta Region and the fashion consumers in the cultural creativity areas. On the basis of the target groups in the exhibition, we have first established the image communication strategy of "We-Media plus Interactive Experience" that focuses on the online promotion of new media. We-Media advantage of WeChat has been fully exerted through "mobile phone (WeChat) plus designated media in and outside the exhibition venue (outdoor electronic screen and guidance logos)". We release information through WeChat Official Account and launch topics and events to attract the extensive attention of the public and the secondary transmission. At the same time, outdoor media and intrafield logos assist to launch an efficient and accurate continuous dissemination and promotion activity. In terms of the overall design of visual identification system, the 6 departments of the School of Art and Design involved this time almost cover all the fields of design disciplines. The content of works on the exhibition is diversified and inclusive. According to the characteristics of the We-Media communication and the application environment of the interaction of screens, when designing graphics, color and standard font, we have formulated the design basis that preferentially considers the digital communication environment and realized the visual extension of guidance logos in the exhibition venue under the internet communication environment through the multi-dimensional and dynamic changeable identification system. Moreover, the content of visual design in the field of new media is added, including the digital invitation for users of WeChat and QQ and the publicity of dynamic videos released on the new media terminals such as WeChat Official Account, official website and outdoor electronic display screen. The theme of this exhibition is "Light Design", which contains the meanings of "organic design", "Minimized Design" and "To Create the Relaxed and Comfortable Attitude towards Life". Meanwhile, it refers in particular to the design of Guangdong Industry Polytechnic and carries out the educational idea followed by the school for a long time. The logo design fully considers the possibility of dynamic extension of visual images. On the basis of taking the simple cube as the basic form, it is expanded to free form of dynamic and changeable combination of graphics. Visual graphics and fonts extracted from the LOGO of the School of Art and Design are interspersed, adhered and folded in the cub. The space and combination forms change and the pattern of cube combination is unfixed. Because the capacity of the whole exhibition is high and complex, considering the difference and connection of different disciplines and specialties of design, we take the main mark as the basic "module" and rearrange and transform through the characteristics that it is changeable and flexible to produce new symbols. At the meantime, new colors are used to differentiate the information modules of different disciplines and specialties. (See "Fig. 6") Besides, we apply it to the design of exhibition materials such as indicators, logos and guidance and build the unified visual environment of the exhibition through the method of visual design of information. Because topic text, environment, time and exhibition media are different, we adopt the corresponding combining forms. Although the mobile, changeable and irregular forms are adopted, it leaves a continuous and overall visual image that is easy to remember on people (see "Fig. 7"). The exhibition opens to the public from June 9 to June 16 and is visited by nearly 50,000 people. It has attracted local audience of Guangzhou and those from Shenzhen, Zhuhai, Foshan and Hong Kong and Macao regions. The degree of popularity among the audiences is beyond our expectation. The visual image design is well received by professional and nonprofessional audiences present in the exhibition and has highlighted the advantage and vitality of the dynamic design of visual images in dissemination. 


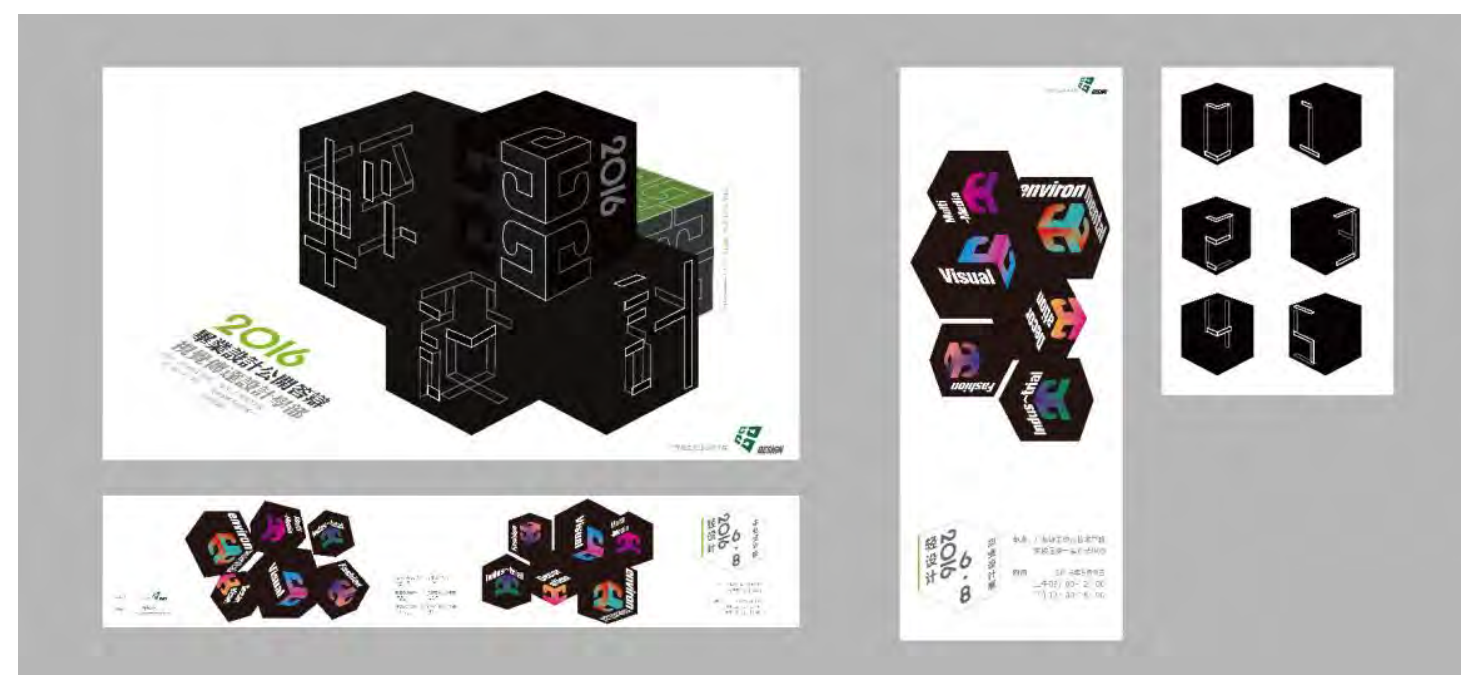

Fig. 6. Visual image design on the graduation design exhibition of Guangdong Industry Polytechnic.

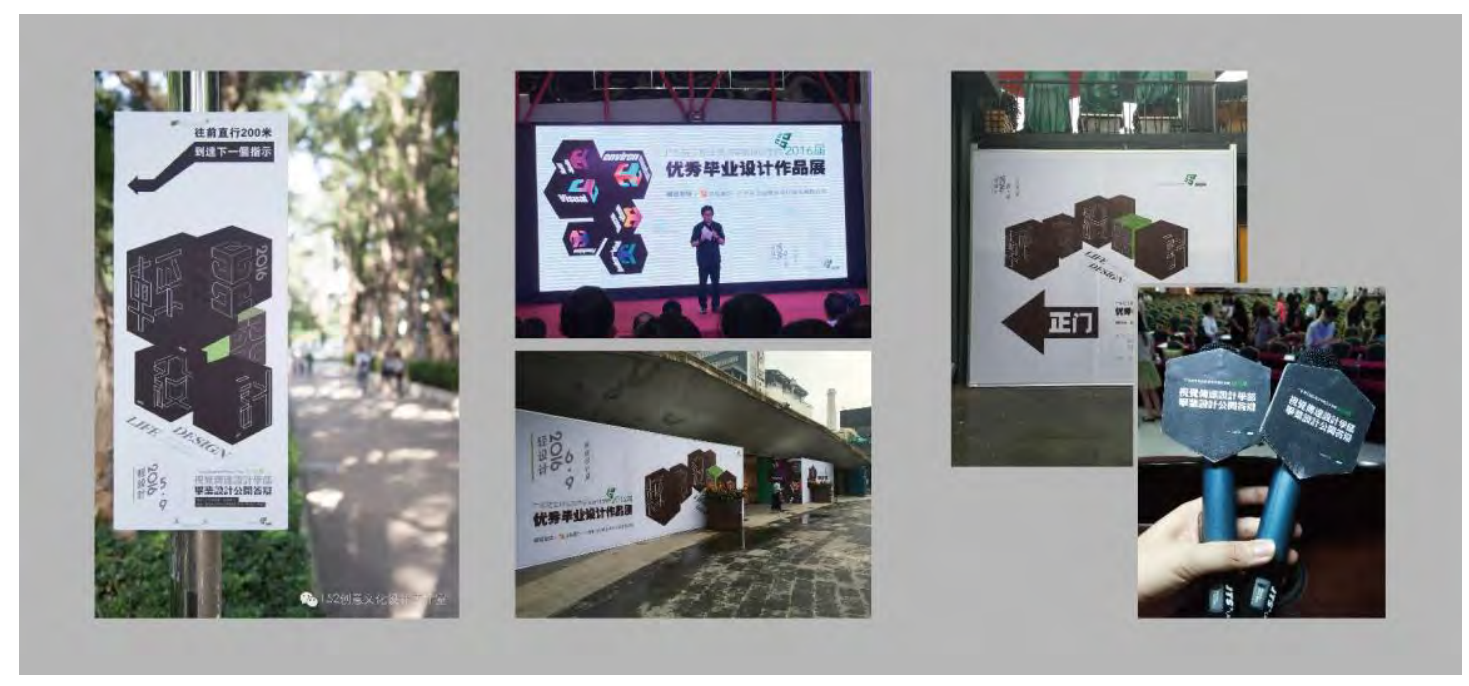

Fig. 7. Application of visual image design on the graduation design exhibition of Guangdong Industry Polytechnic.

\section{CONCLUSION}

Using new media to carry out exhibition promotion and dissemination under the situation of "Internet Plus" has become increasingly popular in this industry. To some degree, new media will become the "source" of thinking on visual image design. For visual communication design, the application of new technology is the inevitable choice of the development of design. Meanwhile, new technology has expanded the connotation and meaning of visual image design on art exhibition and enriched the forms of design. The corresponding aesthetic style and criteria for criticism under the dynamic tendency of the identity design of visual images will be more and more explicit.

\section{REFERENCES}

[1] Ministry of Education, State Language Work Committee, Report on Language Life in China (2016) (in Chinese)
[2] Li Degeng. Qwer Studio [M], Beijing: China Youth Publishing House, 2004. (in Chinese)

[3] Xu Yibing. Innovative Strategy and Practice on the Visual Identity Design of Exhibition under the Dissemination Environment of New Media - Taking the Image Identity Design on the 2016 Shanghai Art and Design Exhibition [J], Decoration, Sept.2016. (in Chinese)

[4] Zhu Qiying. Research on the Dynamic Development of Logo Design [J], Packaging Engineering, Apr.2009. (in Chinese)

[5] Zhang Yali. Dynamic Development in the Design and Promotion of Brand Visual Image - Taking MB (Movingbrands) as an Example [J], Decoration, Mar.2016. (in Chinese) 